\title{
CENTRALIZERS OF IMMERSIONS OF THE CIRCLE
}

\author{
CARLOS ARTEAGA
}

(Communicated by Kenneth R. Meyer)

\begin{abstract}
We prove here that for every element $f$ of an open and dense subset of immersions of the circle $S^{1}$, either the centralizer $Z(f)$ of $f$ is trivial (i.e. $f$ only commutes with its own powers) or $f$ is topologically conjugate to a map $f_{n}: S^{1} \rightarrow S^{1}$ given by $f_{n}(z)=z^{n}$ and, in this case, if $h$ is the conjugacy between $f$ and $f_{n}$ then $Z(f)$ is a subgroup of $\left\{h^{-1} \circ \omega f_{m} \circ h ; m \in \mathbf{N}\right.$ and $\left.\omega^{n-1}=1\right\}$.
\end{abstract}

\section{INTRODUCTION}

Let $\operatorname{Imm}\left(S^{1}\right)$ be the group of $C^{\infty}$ immersions of the circle $S^{1}$ (i.e. maps of $S^{1}$ onto itself without critical points). For $f \in \operatorname{Imm}\left(S^{1}\right), Z(f)$ denotes the centralizer group of $f$, i.e. the set of elements in $\operatorname{Imm}\left(S^{1}\right)$ that commute with $f$. We say that $f$ has trivial centralizer if $Z(f)$ is reduced to the iterates $\left\{f^{n}, n \in N\right\}$ of $f$.

We denote by $f_{n}$ the immersion of $S^{1}$ given by $f_{n}(z)=z^{n}$.

The purpose of this paper is to prove the following result.

Theorem. Let $\operatorname{Imm}\left(S^{1}\right)$ be given the $C^{r}$ topology, $r \in N$. Then there is an open and dense subset $\mathscr{U}$ of $\operatorname{Imm}\left(S^{1}\right)$ such that for $f \in \mathscr{U}$, either $Z(f)$ is trivial or $f$ is topologically conjugate to a map $f_{n}$ and in this case, if $h: S^{1} \rightarrow S^{1}$ is the conjugacy between $f$ and $f_{n}$ then $Z(f)$ is a subgroup of $\left\{h^{-1} \circ \omega f_{m} \circ h ; m \in N\right.$ and $\left.\omega^{n-1}=1\right\}$.

This result is an extension to immersions of a theorem of Kopell [2, Theorem 3 ], who showed the triviality of the centralizer for an open and dense subset of diffeomorphisms of the circle.

The proof of the theorem is based on a result of Mañe [3] that states that structural stability is $C^{r}$ generic in $\operatorname{Imm}\left(S^{1}\right)$.

From the theorem it follows that,

$$
Z\left(f_{n}\right)=\left\{\omega f_{m} ; m \in N, \omega^{n-1}=1\right\} .
$$

Received by the editors July 20, 1989 and, in revised form, September 19, 1989.

1980 Mathematics Subject Classification (1985 Revision). Primary 58F15.

The author was partially supported by CNPq.Brazil. 
Now observe that if an immersion $f: S^{1} \rightarrow S^{1}$ is topologically conjugate to a map $f_{n}$ by a conjugacy $h$, then $f$ commutes with $h^{-1} \circ \omega f_{m} \circ h$ for all $m \in N$ and all $\omega \in S^{1}$ satisfying $\omega^{n-1}=1$. Since $h^{-1} \circ \omega f_{m} \circ h$ is not necessarily $C^{\infty}$, we were not able to prove that

$$
Z(f)=\left\{h^{-1} \circ \omega f_{m} \circ h ; m \in N, \omega^{n-1}=1\right\} .
$$

\section{Proof of the theorem}

We begin by recalling some basic concepts and establishing preliminary results.

Let $f: S^{1} \rightarrow S^{1}$ be a immersion of $S^{1}$. As usual we say that $z \in S^{1}$ is a periodic point of $f$ if $f^{n}(x)=x$ for some $n \in N$. In this case we say that $x$ is a sink if $\left|\left(f^{n}\right)^{\prime}(x)\right|<1$ and a source if $\left|\left(f^{n}\right)^{\prime}(x)\right|>1$. The basin of a sink is defined as the set of points $y$ such that $\lim _{n \rightarrow \infty}\left|f^{n}(x)-f^{n}(y)\right|=0$. It is an open set containing $x$. We denote by $\Sigma(f)$ the complement of the union of the basins of the sinks of $f . \Sigma(f)$ is invariant under both $f$ and $f^{-1}$. We say that $\Sigma(f)$ is hyperbolic if there exist constants $k>0$ and $\lambda>1$ satisfying

$$
\left|\left(f^{n}\right)^{\prime}(x)\right|>k \lambda^{n} \quad \text { for all } x \in \Sigma(f) \text { and } n>0 .
$$

We note that if $h \in Z(f)$ then the set of sinks of $f$ (sources of $f$ ) and the basins of the sinks are invariant under $h$.

The following lemma is basic in the proof of the theorem.

Lemma 2.1. Let $g: S^{1} \rightarrow S^{1}$ be a strictly monotone continuous map of $S^{1}$. If $g \circ f_{k}=f_{k} \circ g$ for some $k>1$ then $g=\omega f_{m}$ where $m=$ degree $g$ and $\omega$ is $a(k-1)$ th root of unity.

Proof. By hypothesis, $g$ satisfies

$$
g\left(z^{k}\right)=g(z)^{k} \quad \text { for all } z \in S^{1} .
$$

Then, if $\omega=f(1)$, we have $\omega^{k-1}=1$ and this implies that $\omega^{-1} g$ commutes with $f_{k}$. Hence we can assume without loss of generality that $g(1)=1$, so we have to show that

$$
g(z)=z^{m} \quad \text { for all } z \in S^{1} .
$$

First we claim that $g$ is order preserving. Otherwise, by hypothesis, there exists a point $z \in S^{1}$ sufficiently close to 1 and such that

$$
1<z<z^{k} \text { and } g\left(z^{k}\right)<g(z)<g(z)^{k}<1 .
$$

This contradicts $(*)$ and proves the claim.

We now consider the inverse image of 1 under $f_{k}$ and $g$. The points mapping to 1 under $f_{k}$ are exactly the $k$ th roots of unity, which can be represented by the points

$$
1<z_{1}<z_{1}^{2}<\cdots<z_{1}^{k-1} \quad \text { where } z_{1}^{k}=1 .
$$


Similarly, $g$ being of degree $m$ and strictly monotone, maps exactly $m$ points

$$
1=p_{0}<\cdots<p_{m-1}
$$

to 1 . We claim that

$$
g\left(z_{1}^{i}\right)=\left(z_{1}^{i}\right)^{m} \quad \text { for all } i=0,1, \ldots k-1 .
$$

In fact, if $i=0$, the claim is obvious because $g(1)=1$. Suppose now, inducting on $i$ that $g\left(z_{1}^{i}\right)=\left(z_{1}^{i}\right)^{m}$. Since $\left[z_{1}^{i}, z_{1}^{i+1}\right)$ maps under $f_{k}$ bijectively to $S^{1}$, there exist exactly $m+1$ points

$$
z_{1}^{i}=x_{0}<x_{1}<\cdots<x_{m}=z_{1}^{i+1}
$$

satisfying $f_{k}\left(x_{j}\right), j=0, \ldots m-1$ and $f\left(x_{m}\right)=1$. This and $(*)$ imply that $g\left(x_{j}\right)^{k}=1$ and so $g\left(x_{j}\right)=z_{1}^{l}$ for some $l=0, \ldots k-1$. We note that between two successive elements $x_{j}, x_{j+1}$ there is no other inverse image of $z_{1}^{l}$ under $g$, because if $g(x)=z_{1}^{l}$ for some $l$, then by $(*)$

$$
g\left(x^{k}\right)=g(x)^{k}=\left(z_{1}^{l}\right)^{k}=1,
$$

so $x^{k}=p_{j}$ for some $0 \leq j \leq m-1$ and this implies that $x=x_{l}$ for some $0 \leq l \leq m$. These properties together with the facts that $g$ is order preserving and $g\left(z_{1}^{i}\right)=\left(z_{1}^{i}\right)^{m}$, imply that $g\left(x_{j}\right)=\left(z_{1}^{i}\right)^{m} z_{1}^{j}$. Hence

$$
g\left(z_{1}^{i+1}\right)=\left(z_{1}^{i}\right)^{m} z_{1}^{m}=\left(z_{1}^{i+1}\right)^{m} .
$$

Similarly, since $g \circ f_{k}^{n}=f_{k}^{n} \circ g$ and $f_{k}^{n}=f_{k^{n}}$ for $n \in N$, we have by the arguments above that $g(z)=z^{m}$ for all inverse images of 1 under $f_{k}^{n}$. Since $\bigcup_{n \in N} f_{k}^{-n}(1)$ is dense in $S^{1}$, we have that $g(z)=z^{m}$ for all $z \in S^{1}$ and the lemma is proved.

Now we prove the theorem. Let

$$
\beta=\left\{f \in \operatorname{Imm}\left(S^{1}\right) ; \Sigma(f) \text { is hyperbolic }\right\} .
$$

It follows from [3] that $\beta$ is open and dense in $\operatorname{Imm}\left(S^{1}\right)$. Let

$$
\begin{aligned}
& \mathscr{U}_{1}=\left\{f \in \beta ; \Sigma(f) \neq S^{1} \text { and } Z(f) \text { is trivial }\right\} \\
& \mathscr{U}_{2}=\left\{f \in \beta ; \Sigma(f)=S^{1}\right\} .
\end{aligned}
$$

By [1, Theorem A], $\mathscr{U}_{2}$ is open in $\operatorname{Imm}\left(S^{1}\right)$ and if $f \in \mathscr{U}_{2}$ then $f$ is topologically conjugate to a map $f_{k}$, for some $k>1$. Let $h: S^{1} \rightarrow S^{1}$ be a conjugacy between $f$ and $f_{k}$. Then, for $g \in Z(f)$ we have that $h \circ g \circ h^{-1}$ is strictly monotone and

$$
\begin{aligned}
\left(h \circ g \circ h^{-1}\right) \circ f_{k} & =h \circ g \circ f \circ h^{-1} \\
& =h \circ f \circ g \circ h^{-1}=\left(h \circ f \circ h^{-1}\right) \circ\left(h \circ g \circ h^{-1}\right) \\
& =f_{k} \circ\left(h \circ g \circ h^{-1}\right) .
\end{aligned}
$$


Thus by Lemma $2.1 h \circ g \circ h^{-1}=\omega f_{m}$ where $m=$ degree $g$ and $\omega$ is a $(k-1)$ th root of unity. Therefore to show the theorem, it is sufficient to show that $\mathscr{U}_{1}$ is open in $\beta$ and dense in $\beta-\mathscr{U}_{2}$.

We shall prove that $\mathscr{U}_{1}$ is dense in $\beta-\mathscr{U}_{2}$ by adapting a technique due to Kopell [2]. Let $f \in \beta-\mathscr{U}_{2}$. If degree $f=1$ then by [2, Theorem 3], $f$ can be arbitrarily approximated by some $\tilde{f} \in \mathscr{U}_{1}$, so we assume degree $f>1$. Since $\Sigma(f)$ is hyperbolic and $S^{1}-\Sigma(f)$ is nonempty, the set of sinks of $f$ is finite and nonempty. Let $p_{0}^{0}, p_{1}^{0}, \ldots, p_{m}^{0}$ be the sinks of $f$ and let $\left(p_{i}^{1}, p_{i}^{2}\right)$ be the component of the basin of $p_{i}^{0}$ containing $p_{i}^{0}, i=0, \ldots m$. Let $F=f^{n}$ be the first iterate of $f$ such that $F\left(p_{i}^{k}\right)=p_{i}^{k}, k=0,1,2$ and $i=0, \ldots m$. By making a small perturbation in $f$, we may assume that $F^{\prime}\left(p_{i}^{k}\right) \neq F^{\prime}\left(p_{j}^{l}\right)$, unless $\exists s \in N$ satisfying $f^{s}\left(p_{i}^{k}\right)=p_{j}^{l}$. By [2, Lemma 5], one can choose a diffeomorphism $\widetilde{F}_{0}:\left[p_{0}^{0}, p_{0}^{2}\right] \rightarrow\left[p_{0}^{0}, p_{0}^{2}\right]$ arbitrarily close to $F /\left[p_{0}^{0}, p_{0}^{2}\right]$ such that $\widetilde{F}_{0}^{\prime}\left(p_{0}^{i}\right)=F^{\prime}\left(p_{0}^{i}\right), i=0,2$ and $Z\left(\widetilde{F}_{0}\right)=\left\{\widetilde{F}_{0}^{n}, n \in \mathbf{Z}\right\}$. Let

$$
\tilde{f} / s^{1}-\left[p_{0}^{0}, p_{0}^{2}\right]=f / s^{1}-\left[p_{0}^{0}, p_{0}^{2}\right]
$$

and

$$
\tilde{f} /\left[p_{0}^{0}, p_{0}^{2}\right]=\varphi \circ \widetilde{F}_{0}
$$

where $\varphi$ is the inverse of $f^{n-1} / f\left[p_{0}^{0}, p_{0}^{2}\right]$. Let $\widetilde{F}=\tilde{f}^{n}$. We will show that $Z(\tilde{f})$ is trivial.

First we note that since degree $\tilde{f}>1$ and $f$ is monotone, $p_{0}^{2}$ is either a limit point of $\Sigma(\tilde{f})$ or an endpoint of some interval $\left(p_{i}^{1}, p_{i}^{2}\right)$ with $p_{i}^{0} \neq p_{0}^{0}$. Hence by adjoining these intervals to $\left(p_{0}^{1}, p_{0}^{2}\right)$ if necessary, we obtain an interval $I=\left(p_{i}^{1}, p_{j}^{2}\right)$ such that $\left(p_{0}^{1}, p_{0}^{2}\right) \subseteq I . \quad \tilde{f}^{l}(I)=I$ for some $l \in N$, and the endpoints of $I$ are limit points of $\Sigma(\tilde{f})$. It follows from the hyperbolicity of $\Sigma(\tilde{f})$ that for every neighborhood $U$ of $\bar{I}, f^{j}(U)=S^{1}$ for some $j \in N$.

Now we claim that $g \in Z(\tilde{f})$ is completely determined by $g\left(p_{0}^{0}\right)$ and $g^{\prime}\left(p_{0}^{0}\right)$. In fact, let $g_{1}, g_{2} \in Z(\tilde{f}), g_{1}\left(p_{0}^{0}\right)=g_{2}\left(p_{0}^{0}\right)$, and $g_{1}^{\prime}\left(p_{0}^{0}\right)=g_{2}^{\prime}\left(p_{0}^{0}\right)$. Since the set of sinks of $\tilde{f}$ (sources of $\tilde{f}$ ) and the basins of the sinks are $g_{i}$-invariant, $g_{1}(I)=g_{2}(I) \neq S^{1}$. Hence we can choose neighborhoods $U_{1}$ and $U$ of $\bar{I}$ with $U_{1} \subset U$ and such that $\widetilde{F} / U$ and $g_{i} / U$ are injective and

$$
\left(g_{2} / U\right)^{-1} \circ g_{1} \circ \widetilde{F}(x)=\widetilde{F} \circ\left(g_{2} / U\right)^{-1} \circ g_{1}(x) \quad \text { for all } x \in U_{1} .
$$

Since $P_{0}^{0}$ is a fixed point of $\left(g_{2} / U\right)^{-1} \circ g_{1}$ and $\left(\left(g_{2} / U\right)^{-1} \circ g_{1}\right)^{\prime}\left(p_{0}^{0}\right)=1$, we have by [2, Lemma $1(b)]$ that

$$
g_{1}(x)=g_{2}(x) \quad \text { for all } x \in U_{1} .
$$

Therefore, using the facts that $\widetilde{F}^{l}\left(U_{1}\right)=S^{1}$ for some $l \in N$, and $\widetilde{F}^{l} \circ g_{i}=$ $g_{i} \circ \widetilde{F}^{l}, i=1,2$, we conclude that $g_{1}=g_{2}$ and the claim is proved. 
Now let $g \in Z(\tilde{f})$. It is easily checked that $\widetilde{F}^{\prime}\left(p_{0}^{0}\right)=\widetilde{F}^{\prime}\left(g\left(p_{0}^{0}\right)\right)$. This together with the fact that $g\left(p_{0}^{0}\right)=p_{i}^{0}$ for some $0 \leq i \leq m$, implies $g\left(p_{0}^{0}\right)=$ $\tilde{f}^{k}\left(p_{0}^{0}\right)$ for some $1 \leq k \leq n$. Since $\widetilde{F}\left(p_{0}^{0}\right)=p_{0}^{0}, g\left(p_{0}^{0}\right)=\tilde{f}^{k} \circ \widetilde{F}^{j}\left(p_{0}^{0}\right)$ for all $j \in N$. Hence, to show that $g$ is a power of $\tilde{f}$, it is sufficient to show that $g^{\prime}\left(p_{0}^{0}\right)=\left(\tilde{f}^{k}\right)^{\prime}\left(p_{0}^{0}\right)\left(\widetilde{F}^{j}\right)^{\prime}\left(p_{0}^{0}\right)$ for some $j \in N$. But, if $\varphi_{1}$ is the inverse of $\tilde{f}^{k} /\left[p_{0}^{0}, p_{0}^{2}\right]$ then $\varphi_{1} \circ g /\left[p_{0}^{0}, p_{0}^{2}\right] \in Z\left(\widetilde{F}_{0}\right)$, so $\left(\varphi_{1} \circ g\right)^{\prime}\left(p_{0}^{0}\right)=\left(\widetilde{F}^{j}\right)^{\prime}\left(p_{0}^{0}\right)$ for some $j \in N$. Hence we are done.

The proof that $\mathscr{U}_{1}$ is open in $\beta$ uses similar arguments and the Lemma 4 of [2].

\section{REFERENCES}

1. M. V. Jacobson, On smooth mappings of the circle into itself, Math. USSR-Sb. 14 (1971), 163-168.

2. N. Kopell, Commuting diffeomorphisms, Global Analysis, Proc. Sympos. Pure Math. 14 (1970), 165-184.

3. R. Mañe, Hyperbolicity, sinks and measure in one dimensional dynamics, Comm. Math. Phys. 100 (1985), 495-524.

Departamento de Matemática, Universidade federal de São Carlos, 13560, São CARLOS-S.P., BRAZIL 